\title{
Hospital Magnético, ¿qué es y cómo funciona?
}

\section{Magnet hospital ¿what is and how it works?}

Lic. Enf. Patricia Domínguez Sánchez•, Mtra. Rosa A. Zarate Grajales••,

Esp. Enf. José Juan Serrano Diosdado•, Lic. Enf. Miguel Arroyo Merino*

\section{Resumen}

Introducción : En el presente documento se aborda la importancia de conocer modelos de atención hospitalaria conocidos como magnéticos, en donde se logra la calidad del cuidado de enfermería y la satisfacción laboral entre otros factores, que se traduce en beneficios para los pacientes, el personal de enfermería y las instituciones. Desarrollo: Se revisan los antecedentes de los hospitales magnéticos, los elementos que se han identificado como esenciales para proporcionar cuidados de calidad de enfermería. Así como los factores que se han relacionado con este tipo de modelo de atención, que ponen de manifiesto. La importancia del rol del personal de enfermería en la atención de los pacientes y los resultados para las organizaciones. Conclusiones: Es necesario conocer estos modelos de atención en nuestro país, generando líneas de investigación que pongan de manifiesto la importancia que tiene contar con elementos necesarios para generar ambientes y entornos seguros y saludables de trabajo.

\section{Abstract}

Introduction: This study highlights the importance of hospital attention models known as magnet, through which, quality of care and nurse satisfaction are achieved, generating benefits for patients, nurses, and institutions. Development: Showing the importance of nurses, high quality care identified elements and magnet attention model factors are addressed from background literature. Conclusion: It is necessary to adopt these models in our country; and therefore, it is necessary to develop research projects aimed to help generate safe and healthy working conditions for nurses.

\section{Palabras}

Clave:

Hospital Cagnético, Atención de Calidad, Cuidado y seguridad. Condicoines de trabajo

\section{Key Words:}

Magnet hospital, Attention Quality, Safe and Healthy Working Conditions. 


\section{INTRODUCCION}

Es responsabilidad tanto de las instituciones como de los profesionales de la salud, identificar por un lado las características del ambiente de trabajo en el que se desarrollan, y por otro asegurar modelos de práctica más favorables para la calidad del cuidado a los pacientes y contribuir a resultados positivos para el personal y la organización.

Por lo anterior se realizó una revisión de estudios reportados en la literatura donde se muestran repercusiones satisfactorias para el paciente y la institución que se basan en la excelencia del cuidado de enfermería los cuales se denominan "hospitales imán o magnéticos".

Un hospital magnético se identifica por su capacidad para contratar y retener enfermeras calificadas, sin embargo a raíz de estudios que se han realizado desde hace más de una década, se ha demostrado que tienen además características y beneficios para los pacientes, el personal de enfermería y para la institución en su conjunto.

Actualmente existe un programa de acreditación y reconocimiento para identificar hospitales magnéticos en Estados Unidos, el cual se basa en indicadores de calidad y estándares para la práctica de enfermería.

Es preciso conocer estos modelos de práctica de enfermería en nuestro país, para ver el impacto que tienen la estructura y contexto de las organizaciones cuando se conforman estrategias basándose en estos modelos que garantizan la calidad de cuidados a los pacientes y se ponga de manifiesto la importancia del rol del profesional de enfermería.

\section{Desarrollo}

Antecedentes

El concepto de hospital magnético fue introducido por primera vez en 1983, se deriva de una política definida por la Academia Americana de Enfermería (AAN) en 1981, ante el fenómeno grave de escasez de personal de enfermería en Estados Unidos, para lo cual se necesitaba identificar las características de la organización que fueran capaces de retener y atraer al personal de enfermería calificado y buscar las mejores condiciones para la práctica profesional. A raíz de este estudio se determinó que la mejor contribución sería realizar investigación de los diferentes factores que intervenían en la creación de hospitales que atraían y retenían profesionales de enfermería y compartir los resultados con otros hospitales, de manera que instrumentarán acciones a fin de poner en marcha en otras instituciones los valores de excelencia que promueve el modelo de hospital magnético. ${ }^{2}$

Elementos identificados como esenciales de los hospitales magnéticos.

En 1988 Kramer y Schmalenberg publicaron los resultados de los hospitales magnéticos, vinculados a las instituciones de excelencia, y se estableció el concepto en la literatura que incluye el elemento más importante que se ha identificado en estas instituciones, la satisfacción laboral y su impacto en la calidad de los cuidados. ${ }^{3}$

Después de esta primera revisión se vio la conveniencia de implementar un programa de reconocimiento de estos centros denominados magnéticos, por lo que en 1994 se estableció el programa de reconocimiento de los hospitales magnéticos conocido como centro de acreditación de enfermeras de Estados Unidos de América (AANCC), los cuales evalúan 14 elementos fundamentales y esenciales para proporcionar atención de excelencia:

1. Calidad del liderazgo de enfermería. Este indicador se explora en todos los niveles de la organización, se transmite y se percibe el apoyo institucional para el personal y el paciente.

2. Estructura organizativa dinámica y sensible a cambios. La organización tiene un sistema productivo $y$ el funcionamiento incluye la toma de decisiones compartida.

3. Estilo de Gestión. Los dirigentes crean un entorno que apoya la participación. Las enfermeras que prestan servicios en puestos de dirección tienen visibilidad y se comunican de manera eficaz con el personal.

4. Programas y políticas de personal. Los salarios y los beneficios de la organización son competitivos. Existe dotación de personal que favorece un ambiente de trabajo saludable y seguro tanto para el paciente como para las enfermeras.

5. Modelos de atención profesional. Las enfermeras son responsables y tienen autonomía de sus propias prácticas, así como de la coordinación de la atención.

6. Calidad de la atención. Las enfermeras líderes son responsables de proporcionar un entorno que influya positivamente en los resultados 
de la calidad de la atención de los pacientes.

7. Mejora de la calidad. La organización cuenta con estructuras y procesos para la medición de la calidad y programas de mejora de la atención y los servicios dentro de la organización.

8. Recursos. La organización prevé una asistencia hospitalaria adecuada, dotada de recursos, apoyo y oportunidades, además de promover la participación de enfermeras en las organizaciones profesionales.

9. Autonomía de enfermería. La enfermería tiene capacidad para evaluar e implementar acciones independientes según proceda, para la atención de los pacientes basada en la competencia, experiencia profesional y el conocimiento.

10. La Comunidad y la organización de salud. La institución establece relaciones entre diferentes organizaciones hospitalarias y organizaciones comunitarias, para desarrollar asociaciones sólidas que apoyen la mejora de los resultados de la salud de los pacientes.

11. Las enfermeras como personal docente. Los Profesionales de enfermería participan en actividades educativas dentro de la organización y la comunidad.

12. Imagen de la enfermería. Los servicios prestados por las enfermeras se caracterizan como esenciales por otros miembros del equipo de atención médica. La enfermera influye de manera efectiva en todos los procesos de atención. 13. Relaciones interdisciplinarias. Este indicador explora las relaciones de trabajo con las diferentes disciplinas, el respeto mutuo se basa en la premisa de que todos los miembros del equipo de atención a la salud son esenciales y contribuyen de manera significativa en el logro de resultados del paciente.

14. Desarrollo profesional. La organización hospitalaria apoya el crecimiento y desarrollo profesional, promueve programas para la educación formal y la certificación profesional.

Para vincular estos elementos fundamentales de los hospitales magnéticos con la capacidad de proporcionar alta calidad de atención a los pacientes, Kramer y Schmalenberg en el 2001, realizaron un estudio

1. Apoyo para la educación

2. Compañeros clínicamente competentes.

3. Relación enfermera-médico

4. Autonomía clínica.

5. Control de la práctica de enfermería.

6. Asignación adecuada de personal.

7. Valores centrados en el paciente.

8. Apoyo administrativo a las funciones de enfermería. con 279 enfermeras que laboraban en hospitales magnéticos, esta encuesta permitió identificar los 8 elementos esenciales para proporcionar atención de calidad, relacionados directamente con la satisfacción en el trabajo (los cuales se denominaron factores esenciales del magnetismo). ${ }^{4}$

También se ha identificado que los hospitales magnéticos se caracterizan por tener una estructura horizontal, con poco personal supervisor, dando a los profesionales de enfermería mayor responsabilidad y autonomía, control sobre la práctica y satisfacción laboral.

El programa de reconocimiento a los hospitales magnéticos desarrollado por el Centro de Acreditación de enfermeras, ha permitido que los investigadores realicen estudios dentro de las instituciones reconocidas como magnéticas, partiendo de las características de los entornos de trabajo (caracterís- 
ticas de la organización), es decir las instituciones que están bajo este modelo, proporcionan los medios y el apoyo de un jefe líder a las enfermeras para utilizar su experiencia y conocimientos de manera que favorezcan las condiciones para otorgar cuidados de excelencia al paciente, lo que aumenta la satisfacción y por tanto mayor retención y menor intención de abandonar los lugares de trabajo. ${ }^{5}$

\section{Factores relacionados con los hospitales magnéticos.}

Los pacientes que son atendidos en hospitales donde se caracterizan por tener suficiente personal de enfermería, apoyo administrativo para la atención de enfermería, buenas relaciones entre enfermeras y médicos se muestran satisfechos con la atención que reciben y las enfermeras reportan menores niveles de desgaste emocional. ${ }^{6}$

Estudios relacionados han reportado que la identificación de factores que contribuyen a crear condiciones para atraer y retener enfermeras calificadas deben ser puestos en marcha por las directoras de enfermería y los administradores de recursos humanos de manera que se pueda rediseñar el trabajo en la práctica de los profesionales de enfermería, contribuyendo a la mejora de la calidad de la atención para los pacientes, la satisfacción de las enfermeras en sus puestos de trabajo, así como a resultados positivos para la organización. ${ }^{7,8}$

Estos resultados están relacionados con la disminución de la mortalidad de los pacientes, principalmente cuando en las instituciones existen factores que ya se han identificado en los hospitales mag- néticos, como el contar con enfermeras de mayor nivel de formación académica, así como una adecuada dotación de personal. ${ }^{9}$

Otros estudios relacionados han identificado la presencia del síndrome de burnout (SB) de manera importante en el personal de enfermería particularmente en los Estados Unidos en donde se ha encontrado que existe un $43 \%$ de nivel de desgaste asociado a la insatisfacción laboral de las enfermeras, también identificaron que la sobrecarga laboral relacionada con el número de pacientes que se asigna por enfermera aumenta la mortalidad. ${ }^{10,9}$

\section{Conclusiones}

La revisión anterior nos permite identificar líneas de investigación que las enfermeras administradoras debemos atender en nuestro país a fin de identificar el fenómeno relacionado con la satisfacción laboral a partir de la identificación de los factores que pueden incidir en la calidad y la satisfacción del personal de enfermería, de manera que permita proponer estrategias para generar ambientes y entornos saludables para el trabajo de enfermería y contender con el impacto en la escasez, el ausentismo y la rotación de las enfermeras, particularmente en la calidad del cuidado de los pacientes y su familia, lo que se traducirá en beneficios para las instituciones del sistema de salud en nuestro país.

\section{REFERENCIAS BIBLIOGRÁFICAS}

Cook A, Hiroz J, Mildon B. Magnet hospitals: characteristics of magnet hospitals fact sheet I of II. Nursing Health Services Research Unit 2006; consultado 12 de enero de
2009. Disponible en: http://www. nhsru.com/factsheets/Magnet\%20 Hospitals\%20Magnet\%20Part\%20 I\%20of\%20II\%20-\%20Characteristics.FINAL.pdf.

McClure M. Magnet hospitals insights and issues. Nurs Adm Q. july/September, 2005; 29 (3):198-201.

Kramer M, Schmalenberg C. Aspectos esenciales del trabajo enfermero en los hospitales magnéticos I. Nursing 2005; 23(3):8-13. Consultado 12 enero de 2009. Disponible en: http://www.doyma.es/revistas/ ctl_servlet?_f=7064\&ip=189.245.2 9.208\&articuloid $=13073039 \& \mathrm{revi}$ staid $=20$

Schmalenberg C. Kramer M. Essentials of a productive nurse work environment. Nurs Res. jan/feb, 2008; 5(1): 2-13.

Upenieks VV. Assesing differences in job satisfaction of nurses in magnet and Nonmagnet hospitals. J Nurs Adm. 2002; 32 (11): 564-76.

Vahey DC, Aiken LH, Sloane DM, Clarke SP, Vargas D. Nurse burnout and patient satisfaction. Med Care. 2004; 42 (2): 57-66.

Laschinger HS, Almost J, Tuer-Hodes D. Workplace empowerment and magnet hospital characteristics. J Nurs Adm. 2003; 33 (7/8):410-22.

Stordeur S, D'Hoore W. Organizational configuration of hospitals succeeding in attracting and retaining nurses. J Adv Nurs. 2006; 45-58

Tourangeau A, Doran DH, McGuillisHall L, Obrien-Pallas L, Pringle $\mathrm{D}, \mathrm{Tu} J \mathrm{~V}$, et al. Impact of hospital nursing care on 30-day mortality for acute medical patients. J Adv Nurs. 2007; 57 (1): 32-44.

Aiken LH, Clarke SP, Sloane DM, Sochalski J, Silber JH. Hospital nurse staffing and patient mortality, nurse burnout, and job dissatisfaction. JAMA. 2002; 288(16):1987-93. 